\title{
EFECTO DE ESTACIÓN DELAÑO SOBRE LA COMPOSICIÓN PROXIMAL Y PERFIL DE ÁCIDOS GRASOS DE CARNE DE LLAMAS EN CRIANZA EXTENSIVA
}

\author{
Effect of Season on Proximate Composition and Fatty Acids Profile of \\ Llama Meat in Extensive Rearing
}

\author{
Lindon W. Mamani-Linares ${ }^{1,3}$, Faustina Cayo ${ }^{2}$, Carmen Gallo ${ }^{1}$
}

\section{Resumen}

\begin{abstract}
Se comparó la composición proximal y perfil de ácidos grasos de carne de llamas faenadas en dos estaciones del año. Se utilizaron 20 llamas de 18 a 24 meses de edad criadas sobre pasturas nativas. Diez animales fueron faenados en la estación seca y 10 en la estación lluviosa bajo procedimientos estándar. Las canales fueron refrigeradas durante $24 \mathrm{~h}$ a $4-6^{\circ} \mathrm{C}$. Se tomaron muestras del músculo Longissimus lumborum (LL) para análisis de humedad, proteína bruta, grasas y cenizas. La carne faenada en la estación lluviosa presentó mayor contenido de grasa intramuscular $(\mathrm{p}<0.10)$ y mayores niveles de ácidos grasos monoinsaturados y poliinsaturados $(\mathrm{p}<0.05)$, pero niveles más bajos de ácidos grasos saturados $(p<0.05)$ en comparación a la carne de llamas faenadas en la estación seca. La relación ácidos grasos poliinsaturados/saturados y el contenido de ácido linoleico conjugado de la carne fue superior en la estación lluviosa. Sin embargo, las carnes de ambas estaciones mostraron la relación de n-6/n-3, dentro de los niveles recomendados (4) y buenos niveles de ácidos grasos deseables (DFA). En conclusión, la carne de llamas jóvenes de ambas estaciones presenta características de grasa favorables para el consumo.
\end{abstract}

Palabras clave: estación del año, ácidos grasos, carne, llama

\section{Abstract}

This study compared the proximate composition and fatty acids profile of llamas slaughtered in two seasons. Twenty 18-24 month old llamas reared in native pastures were used, of which 10 were slaughtered in the dry season and 10 in the rainy season

\footnotetext{
${ }^{1}$ Instituto de Ciencia Animal, Facultad Ciencias Veterinarias, Universidad Austral de Chile, Valdivia, Chile

${ }^{2}$ Universidad Pública de El Alto, La Paz, Bolivia

${ }^{3}$ E-mail: willymlmvzupea_2@hotmail.com
}

Recibido: 14 de marzo de 2013

Aceptado para publicación: 29 de mayo de 2013 
under standard procedures. Carcasses were stored for $24 \mathrm{~h}$ in a cold room (4 to $6^{\circ} \mathrm{C}$ ). Samples of the Longissimus lumborum muscle were collected and moisture, crude protein, lipids and ashes were analysed. Llama meat slaughtered in the rainy season had higher intramuscular fat content $(\mathrm{p}<0.10)$ and higher levels of monounsaturated and polyunsaturated fatty acids $(\mathrm{p}<0.05)$, but lower levels of saturated fatty acids $(\mathrm{p}<0.05)$ as compared to llama meat slaughtered in the dry season. The ratio polyunsaturated/saturated fatty acid ratio and linoleic conjugated content was higher in llama meat of the rainy season. However, meat of both seasons showed an n-6/n-3 ratio within recommended levels (4) and good levels of desirable fatty acids (DFA). In conclusion, the meat of young llamas of both seasons has favorable fat characteristics for consumption.

Key words: season of the year, fatty acids, meat, llama

\section{INTRODUCCIÓN}

El contenido de grasa y el perfil de ácidos grasos en la carne han recibido una creciente atención debido a sus implicancias en la salud humana y la calidad del producto. Diversos estudios epidemiológicos han asociado el consumo de carne roja y carne procesada con el desarrollo de dos de las principales enfermedades crónicas en el mundo occidental, las enfermedades cardiovasculares (ECV) y el cáncer de colon (Giovannucci et al., 1994; Kelemen et al., 2005; Cross et al., 2007; Kontogianni et al., 2008). Algunos constituyentes de las carnes rojas han sido propuestos como responsables de estas asociaciones: el contenido en grasa, los ácidos grasos y la posible formación de compuestos cancerígenos como las aminas heterocíclicas (HCAs) por cocinado de carne a altas temperaturas (Bingham et al., 2002).

Las proporciones de ácidos grasos poliinsaturados (PUFA) con grasas saturadas (SFA) y de omega-6 (n-6) con omega-3 (n-3) PUFA, así como de hipocolesterolémicos con hipercolesterolémicos $(\mathrm{h} / \mathrm{H})$ son ampliamente utilizadas para evaluar el valor nutricional de las grasas. En las últimas décadas, la investigación se ha centrado en los efectos de los ácidos grasos individuales sobre el metabolismo de los lípidos y la prevención de las enfermedades coronarias.
Recientemente, las investigaciones se han centrado en el ácido linoleico conjugado (CLA) debido a su actividad anticancerosa. Evidencia sustancial apoya el papel de la carne roja magra como positivo moderador de los perfiles de lípidos, habiendo estudios recientes que la identifican como una fuente dietética de anti-inflamatorios, los n-3 PUFA de cadena larga (LC) y el CLA (McAfee $e t$ al., 2010).

Se ha demostrado que dietas basadas en pasto pueden mejorar significativamente la composición de los ácidos grasos (FA) y el contenido de antioxidantes de la carne de bovino, aunque con efectos variables sobre la palatabilidad en general. Las dietas basadas en pasto verde mejoran los isómeros totales de CLA (C18: 2), del ácido transvaccénico (TVA) (C18: 1 t11), un precursor de CLA, y de FA como omega-3 (n-3) en un g/g grasa base (Realini et al., 2004).

La composición de ácidos grasos de la carne es influenciada principalmente por la edad, el régimen de alimentación y el genotipo del ganado (Huerta-Leindez et al., 1993; De Smet et al., 2004; Alfaia et al., 2006).

La escasez de estudios sobre valor nutricional de las grasas de carnes de llamas jóvenes motivó este estudio cuyo objetivo fue determinar y comparar el perfil de ácidos grasos de carne de llamas faenadas en la estación seca y lluviosa. 
Cuadro 1. Composición botánica (\%) de la pastura en la época seca y lluviosa en el municipio de Comanche, provincia Pacajes, La Paz

\begin{tabular}{lcc}
\hline Especies & Estación seca & Estación de lluvias \\
\hline Festuca dolichophylla & 36.54 & 24.27 \\
Stipa ichu & 26.36 & 3.22 \\
Festuca orthophylla & 16.54 & 2.65 \\
Muhlenbergia fastigiata & 2.79 & 10.23 \\
Muhlenbergia peruviana & 0.51 & 2.25 \\
Bromus unioloides & 3.03 & 3.45 \\
Calamagrostis vicunarum & 2.10 & 4.51 \\
Calamagrostis antoniana & 0.03 & 0.42 \\
Calamagrostis heterofila & 0.01 & 3.98 \\
Nassella meyeniana & 0.16 & 8.55 \\
Hordeum muticum & -- & 2.22 \\
Poa candamoana & 0.02 & 1.89 \\
Distichlis spp & 0.34 & 1.55 \\
Eleocharis spp & 0.02 & 1.28 \\
Alchemilla pinnata & 0.05 & 11.68 \\
Hypochooeris spp & -- & 0.12 \\
Trifolium amabile & 0.04 & 7.00 \\
Erodium cicutarum & -- & 0.80 \\
Urucarpidium shepardae & -- & 1.20 \\
Adesmia spinosissima & 0.19 & 0.60 \\
Ephedra americana & 0.44 & 0.78 \\
Margiricarpus pinnatus & 2.13 & 2.50 \\
Baccharis boliviensis & 7.83 & 3.15 \\
Parastrephya lepydophylla & 0.87 & 0.30 \\
Otras especies & 0.02 & 1.40 \\
\hline
\end{tabular}

\section{Materiales y Métodos}

\section{Localización del Estudio y Muestras}

El experimento se realizó en una granja localizada en el municipio de Comanche (latitud $16^{\circ} 45^{\prime} 49^{\prime \prime}$ y longitud $\left.68^{\circ} 2^{\prime} 27^{\prime \prime}\right)$, provincia Pacajes, La Paz, Bolivia. Se utilizaron 20 llamas machos enteros de la variedad Kh'ara.
Los animales tenían entre 18 a 24 meses de edad y eran mantenidos sobre pasturas hasta el sacrificio. Las pasturas eran básicamente nativas, siendo Festuca dolichophylla, Stipa ichu, Muhlenbergia spp, Bromus unioloides, Calamagrotis spp y Festuca orthophylla las más frecuentes (Cuadro 1). En el estación de lluvias, no obstante, los pastos verdes y, entre ellos, Trifolium spp fueron más abundantes. La producción de ma- 
teria seca fue de $603 \pm 72$ y de $2140 \pm 122$ $\mathrm{kg} / \mathrm{ha}$ en la estación seca y de lluvias, respectivamente.

El primer grupo $(n=10)$ de llamas fue sacrificado en noviembre de 2011, que corresponde al periodo seco, y el segundo grupo $(n=10)$ fue sacrificado en marzo de 2012, que corresponde al periodo de lluvias. El faenamiento se hizo en el camal de la localidad de Palcoco, provincia Los Andes, La Paz, siguiendo los procedimientos estándares de la empresa comercial. Las canales estuvieron en refrigeración entre 4 y $6{ }^{\circ} \mathrm{C}$ y las muestras de carne fueron colectadas a las $24 \mathrm{~h}$ del sacrificio. Las muestras fueron tomadas del músculo Longissimus dorsi (L1-L6 músculo Longissimus lumborum, LL) del lado izquierdo de la canal y cortadas en bifes de $2.5 \mathrm{~cm}$ de grosor y almacenadas a $-20{ }^{\circ} \mathrm{C}$.

\section{Composición Proximal}

Se determinó humedad, con el método oficial AOAC 950.46 de secado al horno (AOAC, 1996), proteína bruta con el método oficial AOAC 981.10, método Microkjeldahl Gerhardt que determina N en carne (AOAC, 1996), grasa por el método de la norma chilena NCh 1370/3/1970, y cenizas con el método oficial AOAC 920.153, método directo de calcinación en mufla (AOAC, 1996). Las muestras se analizaron en duplicado.

\section{Análisis de Ácidos Grasos}

Los lípidos de la muestra fueron extraídos de acuerdo a Folch et al. (1957). El extracto de lípidos fue convertido a ésteres metílicos de ácidos grasos (FAME) usando la metodología descrita por Hartman y Lago (1973), utilizando un cromatógrafo de gases (GC-2010 Shimadzu AOC-20i auto inyector, AOC-20s auto sampler Shimadzu) y equipado con una columna capilar Rt-2560 (100 m, $0.25 \mathrm{~nm}$ ID y $0.20 \mu \mathrm{m}$ film thickness).

Los ácidos grasos individuales fueron identificados por comparación de sus tiempos de retención con los estándares de la mezcla de ácidos grasos Sulpeco 37 (Sigma Chemical, UK), y que son utilizados como estándares internos. Los ácidos grasos se expresaron como porcentaje del total de ácidos grasos identificados y agrupados como ácidos grasos saturados (SFA), monoinsaturados (MUFA) y ácidos grasos poliinsaturados (PUFA).

\section{Análisis Estadístico}

El análisis estadístico de los datos se realizó a través de una comparación de medias, utilizando una prueba de «t» para muestras independientes con el programa Statistix para Windows v. 8.0.

\section{Resultados y Discusión}

El contenido de humedad, proteína y ceniza en el músculo LL de llamas no fue estadísticamente diferente, mientras que el contenido de grasa fue más alto en llamas faenadas en la estación de lluvias $(\mathrm{p}<0.10)$ que en las faenadas en la estación seca (Cuadro 2). Asimismo, el contenido de grasa en el presente estudio fue inferior (1.56 a 3.51\%) a la descrita para llamas de 2 a 3.5 años de edad (Polidori et al., 2007; Mamani-Linares y Gallo, 2013).

En el Cuadro 3 se presenta el perfil de ácidos grasos de carne en las dos épocas del año, observándose diferencias significativas en los niveles de SFA, MUFA y PUFA $(\mathrm{p}<0.05)$. En general, los principales ácidos grasos en la grasa intramuscular fueron C18:1n-9, seguido por C16:0 y C18:0 (Cuadro 4).

La época del año tiene un efecto sobre todos los ácidos grasos excepto en C8:0, C17:0, C18:0, C16:1, C18:2n-6, C20:3n-3 y C20:5n-3 (Cuadro 4). Además, la época del año afectó las relaciones de h/H y PUFA/ SFA, y las proporciones de $\sum \mathrm{h}, \Sigma \mathrm{H}, \Sigma \mathrm{CLAy}$ $\sum$ DFA (Cuadro 3). Estos resultados concuerdan con los hallazgos en otros estudios (Santos-Silva et al., 2002; Nürnberg et al., 2008). 
Cuadro 2. Composición proximal (promedio \pm d.e.) del músculo Longissimus lumborum de llamas (Lama glama) criadas en el municipio de Comanche, provincia Pacajes, La Paz y faenadas en dos épocas del año

\begin{tabular}{lcc}
\hline Características & Estación seca & Estación de lluvias \\
\hline Humedad (\%) & $72.58 \pm 0.56^{\mathrm{a}}$ & $72.80 \pm 0.45^{\mathrm{a}}$ \\
Grasa (\%) & $0.42 \pm 0.06^{\mathrm{b}}$ & $0.72 \pm 0.02^{\mathrm{a}}$ \\
Proteína (\%) & $25.19 \pm 0.92^{\mathrm{a}}$ & $24.90 \pm 0.37^{\mathrm{a}}$ \\
Ceniza (\%) & $1.35 \pm 0.05^{\mathrm{a}}$ & $1.32 \pm 0.06^{\mathrm{a}}$ \\
\hline
\end{tabular}

${ }^{a b}$ Superíndices diferentes dentro de filas indican diferencia significativa $(p<0.10)$

Cuadro 3. Índices de ácidos grasos relacionados a la salud humana del músculo Longissimus lumborum de llamas criadas en el municipio de Comanche, provincia Pacajes, La Paz y faenadas en dos épocas del año

\begin{tabular}{|c|c|c|}
\hline Suma e índices ${ }^{1}$ & Estación seca & Estación de lluvias \\
\hline$\sum$ SFA & $47.94 \pm 3.11^{\mathrm{a}}$ & $41.79 \pm 3.37^{\mathrm{b}}$ \\
\hline$\Sigma$ MUFA & $43.50 \pm 1.36^{\mathrm{a}}$ & $48.61 \pm 2.20^{\mathrm{b}}$ \\
\hline$\sum$ PUFA & $8.56 \pm 2.26^{\mathrm{a}}$ & $9.61 \pm 1.76^{\mathrm{b}}$ \\
\hline$\Sigma$ CLA & $1.33 \pm 0.15^{\mathrm{a}}$ & $1.90 \pm 0.21^{\mathrm{b}}$ \\
\hline$\sum \mathrm{n}-3$ & $2.58 \pm 0.48^{\mathrm{a}}$ & $2.80 \pm 0.76^{\mathrm{a}}$ \\
\hline$\sum \mathrm{n}-6$ & $5.98 \pm 1.86^{\mathrm{a}}$ & $6.74 \pm 1.10^{\mathrm{a}}$ \\
\hline$\sum \mathrm{h}$ & $40.93 \pm 3.88^{\mathrm{a}}$ & $48.63 \pm 4.10^{\mathrm{b}}$ \\
\hline$\sum \mathrm{H}$ & $32.98 \pm 2.72^{\mathrm{a}}$ & $27.34 \pm 3.34^{\mathrm{b}}$ \\
\hline$n-6 / n-3$ & $2.30 \pm 0.41^{\mathrm{a}}$ & $2.48 \pm 0.57^{\mathrm{a}}$ \\
\hline $\mathrm{h} / \mathrm{H}$ & $1.24 \pm 0.23^{\mathrm{a}}$ & $1.80 \pm 0.35^{\mathrm{b}}$ \\
\hline PUFA/SFA & $0.18 \pm 0.06^{\mathrm{a}}$ & $0.23 \pm 0.06^{\mathrm{b}}$ \\
\hline$\sum \mathrm{DFA}$ & $63.78 \pm 3.11^{\mathrm{a}}$ & $69.90 \pm 3.75^{\mathrm{b}}$ \\
\hline
\end{tabular}

$a, b$ Superíndices diferentes dentro de filas indican diferencia significativa $(p<0.05)$

${ }^{1}$ SFA: Ácidos grasos saturados, MUFA: ácidos grasos monoinsaturados, PUFA: ácidos grasos poliinsaturados, CLA: ácido linoleico conjugado, n-3: omega 3, n-6: omega 6 , h: hipocolesterolaémicos (suma de 18:1, 18:2, 18:3, 20:5, 22:5, 22:6), H: hipercolesterolaémicos (suma de 12:0, 14:0, 16:0), DFA: ácidos grasos deseables (suma de MUFA+PUFA+C18:0)

En general, animales finalizados sobre forraje verde (faenados en la estación de lluvias) tienen niveles de PUFA, ácidos grasos n-3 y CLA más altos que aquellos finalizados en la estación seca. De acuerdo a Nürnberg et al. (2005) y Realini et al. (2004) los animales finalizados con forraje verde presentan mayores niveles de PUFA, n-3 y CLA 
Cuadro 4. Perfil de ácidos grasos (promedio \pm d.e.) del músculo Longissimus lumborum de llamas criadas en el municipio de Comanche, provincia Pacajes, La Paz y faenadas dos épocas del año

\begin{tabular}{|c|c|c|c|}
\hline Ácidos grasos & & Estación seca & Estación de lluvias \\
\hline \multicolumn{4}{|l|}{ Ácidos grasos saturados } \\
\hline Ac. Caprílico & C 8:0 & $0.10 \pm 0.02^{\mathrm{a}}$ & $0.14 \pm 0.05^{\mathrm{a}}$ \\
\hline Ac. Caprico & C 10:0 & $0.54 \pm 0.12^{\mathrm{a}}$ & $0.82 \pm 0.11^{\mathrm{b}}$ \\
\hline Ac. Láurico & C $12: 0$ & $0.64 \pm 0.14^{\mathrm{a}}$ & $0.41 \pm 0.07^{\mathrm{b}}$ \\
\hline Ac. Tridecanoico & C 13:0 & $0.45 \pm 0.02^{\mathrm{a}}$ & $0.27 \pm 0.01^{\mathrm{b}}$ \\
\hline Ac. Mirístico & C 14:0 & $5.19 \pm 0.91^{\mathrm{c}}$ & $4.26 \pm 1.28^{\mathrm{d}}$ \\
\hline Ac. Pentadecanoico & C $15: 0$ & $1.62 \pm 0.20^{\mathrm{a}}$ & $0.88 \pm 0.18^{\mathrm{b}}$ \\
\hline Ac. Palmítico & C 16:0 & $27.15 \pm 1.82^{\mathrm{a}}$ & $22.66 \pm 1.99^{b}$ \\
\hline Ac. Margarico & C $17: 0$ & $0.61 \pm 0.13^{\mathrm{a}}$ & $0.67 \pm 0.24^{\mathrm{a}}$ \\
\hline Ac. Esteárico & C 18:0 & $11.73 \pm 1.65^{\mathrm{a}}$ & $11.69 \pm 0.90^{\mathrm{a}}$ \\
\hline \multicolumn{4}{|l|}{ Ácidos grasos monoinsaturados } \\
\hline Ac. Miristoleico & C $14: 1 n-5$ & $2.71 \pm 0.22^{\mathrm{a}}$ & $1.56 \pm 0.17^{\mathrm{b}}$ \\
\hline Ac. Penta decaenoico & C $15: 1 n-5$ & $0.50 \pm 0.06^{\mathrm{c}}$ & $0.31 \pm 0.02^{\mathrm{d}}$ \\
\hline Ac. Palmitoleico & C $16: 1 n-7$ & $5.97 \pm 0.28^{\mathrm{c}}$ & $6.76 \pm 0.33^{\mathrm{d}}$ \\
\hline Ac. Palmitoleico & C $16: 1$ & $1.11 \pm 0.35^{\mathrm{a}}$ & $1.29 \pm 0.18^{\mathrm{a}}$ \\
\hline Ac. Heptadecenoico & C $17: 1$ & $0.62 \pm 0.13^{\mathrm{a}}$ & $0.46 \pm 0.20^{\mathrm{b}}$ \\
\hline Ac. Elaídico (trans 9) & C $18: 1$ & $2.56 \pm 0.11^{\mathrm{a}}$ & $3.58 \pm 0.09^{\mathrm{b}}$ \\
\hline Ac. Vaccénico & C $18: 1 \mathrm{n}-9$ & $29.76 \pm 2.0^{\mathrm{a}}$ & $35.64 \pm 2.71^{\mathrm{b}}$ \\
\hline Ac. Eicosenoico & C 20:1 n-9 & $0.43 \pm 0.05^{\mathrm{a}}$ & $0.16 \pm 0.04^{b}$ \\
\hline \multicolumn{4}{|l|}{ Ácidos grasos poliinsaturados } \\
\hline Ac. Linoleico (cis-9 cis-12) & C $18: 2 n-6$ & $5.78 \pm 1.76^{\mathrm{a}}$ & $5.60 \pm 1.17^{\mathrm{a}}$ \\
\hline $\begin{array}{l}\text { Ac. Linoleico (cis-9 trans- } \\
\text { 11) }\end{array}$ & C $18: 2 n-3$ & $0.33 \pm 0.01^{\mathrm{a}}$ & $0.88 \pm 0.02^{\mathrm{b}}$ \\
\hline $\begin{array}{l}\text { Ac. alfa Linolénico (cis-9 } \\
\text { cis-12 cis-15) }\end{array}$ & C $18: 3 n-3$ & $1.00 \pm 0.02^{\mathrm{c}}$ & $1.22 \pm 0.03^{\mathrm{d}}$ \\
\hline Ac. Eicosadienoico & C $20: 2 n-6$ & $0.32 \pm 0.13^{\mathrm{a}}$ & $0.19 \pm 0.04^{b}$ \\
\hline Ac. Eicosatrienoico & C $20: 3 n-3$ & $0.68 \pm 0.26^{\mathrm{a}}$ & $0.68 \pm 0.20^{\mathrm{a}}$ \\
\hline Ac. Eicosapentaenoico & C $20: 5 n-3$ & $0.41 \pm 0.08^{\mathrm{a}}$ & $0.45 \pm 0.09^{\mathrm{a}}$ \\
\hline Ac. Docosapentaenoico & C $22: 5 n-3$ & nd & $0.11 \pm 0.09$ \\
\hline
\end{tabular}

${ }^{a, b}$ Superíndices diferentes dentro de filas indican diferencia significativa $(p<0.05)$

${ }^{c, d}$ Superíndices diferentes dentro de filas indican diferencia significativa $(p<0.10)$

que los finalizados sobre concentrado, proporcionando carne con un perfil de ácidos grasos más favorable desde una perspectiva de salud para el consumidor (Scollan et al., 2006). Los lípidos de forrajes frescos están caracterizados por una predominancia de C18:3, precursor de la serie n-3; mientras que los concentrados contienen niveles relativamente altos de $\mathrm{C} 18: 2$, precursor de la serie n-6 (Fisher et al., 2000; Velasco et al., 2001). 
Grasas que presentan una baja relación PUFA/SFA son consideradas no favorables porque pueden inducir un incremento de colesterol en la sangre. La grasa de rumiantes, especialmente cuando son criados sobre pasturas, presentan valores de PUFA/SFA entre 0.15 a 0.25 , que son valores dentro de lo recomendado (Lee et al., 2008; Dierking et al., 2010). La relación PUFA/SFA de la grasa intramuscular (IMF) de llamas de ambos periodos fue más baja que el mínimo recomendado (0.4), mientras que la relación n-6:n-3 está dentro de la relación dietaría recomendada (debajo de 4) (BDH, 1994). Una relación n-6/n-3 más baja es deseada para reducir el riesgo de las enfermedades crónicas; sin embargo, del total de los ácidos grasos encontrados en la carne de llamas faenadas en el periodo de lluvias (Cuadro 4), solo el $30.1 \%$ es considerada perjudicial para la salud, mientras que en los faenados en periodo seco es similar a las concentraciones encontradas en el músculo Longissimus de bovinos $(36.2 \%)$, pero más bajo que en ovinos (38.42\%) (Göncü Karakök et al., 2010).

\section{Conclusiones}

- La carne de la estación seca presenta niveles más bajos de grasa intramuscular, mientras que el perfil de ácidos grasos de la carne de la estación lluviosa tiene mayores niveles de grasas poliinsaturadas y CLA.

- Las grasas de llamas faenadas en la estación de lluvias tienen niveles más bajos de ácidos grasos perjudiciales para la salud.

- La carne de llamas jóvenes de ambas estaciones presenta características de grasa favorables para el consumo.

\section{Literatura Citada}

1. Alfaia CMM, Ribeiro VSS, Lourenço MRA, Quaresma MAG, Martins SIV, Portugal APV. 2006. Fatty acid composition, conjugated linoleic acid isomers and cholesterol in beef from crossbred bullocks intensively produced and from Alentejana purebred bullocks reared according to Carnalentejana-PDO specifications. Meat Sci 72: 425-436.

2. AOAC. 1996. Official methods of analysis. $16^{\text {th }}$ ed. Washington DC: Association of Analytical Chemist. 1298 p.

3. [BDH] British Department of Health. 1994. Nutritional aspects of cardiovascular disease. Report on health and social subjects. No. 46. London: Her Majesty's Stationery Office. $48 \mathrm{p}$.

4. Bingham SA, Hughes $R$, Cross AJ. 2002. Effect of white versus red meat on endogenous $\mathrm{N}$-nitrosation in the human colon and further evidence of a dose response. J Nutr 132: 3522-3525.

5. Cross AJ, Leitzmann MF, Gail MH, Hollenbeck AR, Schatzkin A, Sinha R. 2007. A prospective study of red and processed meat intake in relation to cancer risk. PLos Med 4(12): e325. doi:10.1371/journal. pmed.0040325.

6. De Smet S, Raes K, Demeyer D. 2004. Meat fatty acid composition as affected by fatness and genetic factors: a review. Animal Res 53: 81-98.

7. Dierking $R$, Kallenbach $R$, Grün $I$. 2010. Effect of forage species on fatty acid content and performance of pasture finished steers. Meat Sci 85: 597-605.

8. Fisher AV, Enser M, Richardson RI, Wood JD, Nute GR, Kurt E. 2000. Fatty acid composition and eating quality of lamb types derived from four diverse breed production systems. Meat Sci 55: 141-147.

9. Folch J, Lees M, Stanley S. 1957. A simple method for the isolation and purification of total lipids from animal tissues. J Biol Chem 226: 497-509.

10. Giovannucci E, Rimm EB, Stampfer MJ, Colditz GA, Ascherio A, Willet WC. 1994. Intake of fat, meat and fiber in relation to risk of colon cancer in men. Cancer Res 54: 2390-2397.

11. Göncü Karakök S, Ozogul Y, Saler M, Ozogul F. 2010. Proximate analysis. Fatty acid profiles and mineral contents 
of meats: a comparative study. J Muscle Foods 21: 210-223.

12. Hartman L, Lago R. 1973. Rapid preparation of methyl esters from lipids. Lab Pract 22: 475-476.

13. Huerta-Leindez NO, Cross HR, Savell JW, Lunt DK, Baker JF, Pelton LS. 1993. Comparison of the fatty acid composition of subcutaneous adipose tissue from mature Brahman and Hereford cow. J Anim Sci 71: 625-630.

14. Kelemen LE, Kushi LH, Jacobs DR, Cerhan JR. 2005. Associations of dietary protein with disease and mortality in a prospective study of postmenopausal women. Am J Epidemiol 161: 239-249.

15. Kontogianni MD, Panagiotakos DB, Pitsavos C, Chrysohoou C, Stefanadis C. 2008. Relationship between meat intake and the development of acute coronary syndromes: The CARDIO2000 case-control study. Eur J Clin Nutr 62: 171-177.

16. Lee J, Kannan G, Eega K, Kouakou $B, G e t z, W .2008$. Nutritional and quality characteristics of meat from goats and lambs finished under identical dietary regime. Small Rum Res 74: 255-259.

17. Mamani-Linares LW, Gallo CB. 2013. Meat quality attributes of the Longissimus lumborum muscle of the Kh'ara genotype of llama (Lama glama) reared extensively in northern Chile. Meat Sci 94: 89-94.

18. McAfee A, McSorley E, Cuskelly G, Moss B, Wallace J, Bonham M, Fearon A. 2010. Red meat consumption: an overview of the risks and benefits. Meat Sci 84: 1-13.

19. Nürnberg K, Dannerberger D, Nürnberg G, Ender K, Voigt J, Scollan ND. 2005. Effect of grass-based and concentrate feeding system on meat quality characteristics and fatty acid composition of longissimus muscle in different cattle breeds. Livest Prod Sci 94:137-147.

20. Nürnberg K, Fischer A, Nuernberg $G$, Ender K, Dannenberger D. 2008. Meat quality and fatty acid composition of lipids in muscle and fatty tissue of Skudde lambs fed grass versus concentrate. Small Rum Res 74: 279283.

21. Polidori P, Renieri C, Antonini M, Passamonti P, Pucciarelli F. 2007. Meat fatty acid composition of llama (Lama glama) reared in the Andean highlands. Meat Sci 75: 356-358.

22. Realini CE, Duckett SK, Brito GW, Dalla Rizza M, De Mattos D. 2004. Effect of pasture vs. concentrate feeding with or without antioxidants on carcass characteristics, fatty acid composition, and quality of Uruguayan beef. Meat Sci 66:567-577.

23. Santos-Silva J, Bessa RJB, SantosSilva F. 2002. Effect of genotype, feeding system and slaughter weight on the quality of light lambs II. Fatty acid composition of meat. Livest Prod Sci 77: 187-194.

24. Scollan N, Hocquette JF, Nuernberg $K$, Dannenberger D, Richardson I, Moloney A. 2006. Innovations in beef production systems that enhance the nutritional and health value of beef lipids and their relationship with meat quality. Meat Sci 74: 17-33.

25. Velasco, S, Cañeque V, Pérez $C$, Lauzurica S, Díaz MT, Huidobro F, et al. 2001. Fatty acid composition of adipose depots of suckling lambs raised under different production systems. Meat Sci 59: 325-333. 